\title{
The Effect of Isometric and Isotonic Exercises of Lower Limbs Extensor Sling on Static and Dynamic Balance in Basketball Players with Patellofemoral Pain
}

\author{
Seyedehzahra Salamifar, Mohammad Hossein Nasermeli*, Behnaz Ganji Namin \\ Department of Physical Education and Sports Sciences, Karaj Branch, Islamic Azad University, Karaj, Iran \\ Email: zahra.salamifar@gmail.com,mh.naser.m@gmail.com,behnezganji@yahoo.ocm
}

How to cite this paper: Salamifar, S., Nasermeli, M.H. and Ganji Namin, B. (2017) The Effect of Isometric and Isotonic Exercises of Lower Limbs Extensor Sling on Static and Dynamic Balance in Basketball Players with Patellofemoral Pain. Health, 9, 1355-1366.

https://doi.org/10.4236/health.2017.910099

Received: May 26, 2017

Accepted: September 19, 2017

Published: September 22, 2017

Copyright $\odot 2017$ by authors and Scientific Research Publishing Inc. This work is licensed under the Creative Commons Attribution International License (CC BY 4.0).

http://creativecommons.org/licenses/by/4.0/

\begin{tabular}{|c|}
\hline (c) \\
\hline
\end{tabular}

\begin{abstract}
Background: Patellofemoral Pain Syndrome (PFPS) is one of the most common conditions among athletes. Although there are various causes for this condition, reduced balance is the problem most frequently expressed by these patients. The aim of the present study was to examine the effect of Extensor Muscle Sling Isometric and Isotonic (EMSII) exercises of lower limbs on female basketball players suffering from PFPS. Method: A total of 30 participants, all female basketball players suffering from PFPS, with an average age of 20 - 30 years, were divided into two isometric/isotonic and control experimental groups. Eight weeks of isometric and isotonic exercises were recommended to the participants with a focus on EMSII exercises of lower limbs in two four-week phases. The control group received no specific exercise program. Pre-test and post-test included the use of Stork balance test (SBT) and Y Balance tests (YBT) to assess the static and dynamic balance, respectively. Results: After eight weeks of performing separate isometric and isotonic exercises on the mentioned muscles, and according to the results of the pre-test, a significant increase was observed in the results of both static and dynamic balance tests $(P<0.05)$. Conclusion: EMSII exercises of lower limbs showed a significant effect on the increased balance. Recommended exercises can be suggested according to the type of exercises as a factor for increasing static and dynamic balance.
\end{abstract}

\section{Keywords}

Isometric Exercises, Isotonic Exercises, Muscle Slings, Patellofemoral Pain Syndrome 


\section{Introduction}

The knee joint is one of the most important components of the motion chain which is directly involved in the movements and forces from the soles of the feet, ankles, and legs. This joint also transfers the forces applied to the hip, pelvis, and spine [1]. Sports such as basketball, volleyball and the likes involve frequent jumps and landings. Most of these powerful and harmonious movements are caused by flexion and extension of the knee in different degrees of motion. Falling down, a direct impact on the knee caused by an opponent's body, knee injuries caused by sudden jumps and maneuvers in order to dodge the opponent, these could all cause significant pressure or strain on the knee joint and its related muscles. The knee joint is therefore one of the most vulnerable joints in the body [2] [3]. That is also why Patellofemoral Pain Syndrome (PFPS) is one of the most common knee injuries among athletes. This syndrome is not easy to diagnose and the diagnosis could be challenging for clinicians [3] [4]. Although sports medicine specialists frequently treat patients with PFPS, pathological origin of this injury is not known yet [5] [6] [7]. Most treatments are costly and invasive and are not prescribed for all patients [4] [5] [8] [9]. Principal solutions to patellofemoral problems are often mechanical. They include lower limb exercises, stretching, use of tape, as well as leg braces and orthoses. In cases where physiotherapy is not beneficial, various surgical procedures are recommended which aim at restoring the natural alignment of the joint. The surgical techniques are not practical for all patients due to their high costs and invasive nature [10] [11] [12]. Therefore, an inexpensive and non-invasive alternative could be significant [9] [13]. Although there is abundant research on the risk factors associated with PFPS, few studies have been devoted to exercise therapy for treating this syndrome. Exercising practices consist of the use of Extensor Muscle Isometric and Isotonic (EMSII) for lower limbs. Little research has been conducted on the impact of EMSII exercises (Gluteus Maximus, Rectus Femoris, and Gastrocnemius) on the balance of these individuals [14]. Like Upper Limb Muscles Slings, Lower Limb Muscle Slings have been designed for simultaneous combined movements of the limbs. In comparison with the synergist muscles which act locally as a single unit to isolate joint movement, muscle slings provide stability around all joints and act globally. Muscle Slings act to facilitate rotation and transmission of forces applied to the body, especially from lower limbs to upper limbs. They also provide mutual stable movement toward antagonist muscles, as it is the case in transitional movements [15].

Maintaining balance is one of the major problems that patients with PFPS face [16] [17] [18]. Certain knee abnormalities such as anterior knee pain syndrome, lead to proprioceptive sensory system failure [19] [20]. Ebrahimi E et al., (2007) reported that proprioception is reduced in patients with anterior knee pain syndrome compared to healthy people [16]. Alterations in the control system can be associated with increased postural sway and decreased balance [21]. Currently, there exist few studies regarding the balance in patients with anterior knee pain. 
In a study conducted in 2009 by Goto et al., the impact of anterior knee pain syndrome on dynamic balance was evaluated in the Star Excursion Balance Test (SEBT) directions. The results showed that in the anterior direction, research subjects were able to travel a shorter distance compared to healthy people. Therefore, participants suffering from this syndrome showed reduced dynamic balance condition compared to healthy individuals [17]. Citake and Yokse (2011) studied the relationship between single-leg balance test and PFPS. The results indicated that there is a significant difference in static single-leg balance in the standing position [22]. In a study, Ebersole et al., (2008) examined the impact of PFPS on an individual's performance in SEBT. The results showed that patients with PFPS had a smaller reaching distance in the posterior direction, compared with healthy individuals [18].

The foregoing discussion demonstrated that, there is no quality study evaluating the effect of EMSII of lower limbs on the static and dynamic balance of basketball players with PFPS, especially in female basketball players. Consequently, the purpose of the present study is to examine the effect of these exercises on the static and dynamic balance of female basketball players suffering from PFPS.

\section{Methods}

This study is a quasi-experimental research with pre-test and post-test, as well as a control group. The study was conducted using the random sampling method. The statistical population of the study consisted of 30 female basketball players with PFPS with an age range of 20 - 30 years. Participants were selected based on the following inclusion criteria: minimum three years of athletic background, positive Patellar Grind test result, diagnosis by a sports medicine specialist. The exclusion criteria were as follows: history of femur, leg and knee fracture, musculoskeletal disorders in the lower limbs, meniscus lesions or severe ligaments damages, osteoarthritis, previous knee surgery, patella tendon injuries, Osgood-Schlatter or lumbar spine injuries. Participants were divided into three isometric $(n=10)$, isotonic $(n=10)$ and control $(n=10)$ groups.

A week before the study, participants were briefed on the exercise protocol and completed the consent forms. Their demographic characteristics were measured at the same time. Twenty-four hours before the exercise, after a four-minute jogging and stretching exercise, and a two-minute break, static and dynamic balance tests were administered.

Since $20 \%$ to $40 \%$ of the time is spent walking on one leg, the static and dynamic balance was assessed on the dominant leg. To assess Stork Balance Test (SBT) $($ ICC $=0.87)$ as a static test, participants were placed on a hard surface while blindfolded. Then their non-dominant leg was lifted out and kept close to the knee. Hands were placed on the iliac crest of pelvis [23]. The time during which each participant could keep this position was recorded by stopwatch and was considered as an indicator of the participant's performance in the balance test. Each participant took the test three times and her highest score (the time 
spent in the above-mentioned position) was calculated. Errors which interrupted the test included detaching of the hands from the iliac crest of the pelvis, removing of the leg from the top of opposite knee, opening the eyes, and any movement of the leg acting as a fulcrum [24].

Y Balance Test (YBT) with ICC $=0.88$ was used to assess the dynamic postural control [25]. Anterior direction makes a 135-degree angle with two other directions, and the two directions of anterior-posterior and posterior-exterior from a 90-degree angle with each other [25]. To conduct the test, the actual leg length from the anterior superior iliac spine (ASIS) to the medial malleolus, was measured in centimeters to normalize the data. Each participant stood in the center on the dominant leg (single-leg) whilst simultaneously reaching as far as possible with the other leg in three separate directions: anterior, posterolateral, and posteromedial. The YBT composite score is calculated by the examiner, to the extent that no error occurred (the leg did not move from the center, no pressure was placed on the leg acting the reaching function and the participant did not lose her balance), and then she returned to the normal position on two legs. The distance between the contact point and the center of $Y$ shape was considered as the reaching distance. Each participant repeated the Y Balance Test three times in each direction at the end; the mean was calculated for each of the three directions. The mean was calculated using the following equation:

$$
\text { Score }=\frac{\text { Fetching distance }}{\text { Leg length }} \times 100
$$

\subsection{Exercise Protocol}

Exercises were administered for a period of 8 weeks, two sessions per week (Table 1 \& Table 2). Given that the highest effect on PFPS exercises is 2 to 4 sets, and 10 reps, the program was designed in two four-week phases with the gradual progression difference [26]. The Extensor Muscle Sling exercises are few in number. There are also very few exercises targeting both PFPS and Extensor Muscle Sling. For this reason, most of the research articles on PFPS recommend 10 seconds of muscular contraction, duration which has been implemented in our research as well [26] [27].

Table 1. Exercise protocol.

\begin{tabular}{|c|c|c|c|c|}
\hline \multicolumn{4}{|c|}{ Isometric Exercises } & \\
\hline & & \multicolumn{2}{|c|}{ Duration } & \\
\hline & & $\begin{array}{l}\text { Phase } 1-4 \\
\text { weeks }\end{array}$ & $\begin{array}{c}\text { Phase } 4 \text { - } 8 \\
\text { weeks }\end{array}$ & \\
\hline Exercise & Tempo & Volume & Volume & Rest/hold \\
\hline Wall squat $45^{\circ}$ & slow & $2 \times 10$ second & $4 \times 10$ second & $\begin{array}{l}10 \text { second hold/5 } \\
\text { second rest }\end{array}$ \\
\hline Jumping from step in pairs & slow & $2 \times 10$ second & $4 \times 10$ second & $\begin{array}{l}10 \text { second hold } / 5 \\
\text { second rest }\end{array}$ \\
\hline
\end{tabular}




\section{Continued}

\begin{tabular}{|c|c|c|c|c|}
\hline $\begin{array}{c}\text { Hip and knee flexion/extension } \\
\text { using partner }\end{array}$ & slow & $2 \times 10$ second & $4 \times 10$ second & $\begin{array}{l}10 \text { second hold } / 5 \\
\text { second rest }\end{array}$ \\
\hline $\begin{array}{l}\text { Supine pushing the ball against the } \\
\text { wall, pushing the tiller }\end{array}$ & slow & $2 \times 10$ second & $4 \times 10$ second & $\begin{array}{l}10 \text { second hold } / 5 \\
\text { second rest }\end{array}$ \\
\hline Lunges $0^{\circ}-45^{\circ}$ flexion & slow & $2 \times 10$ second & $4 \times 10$ second & $\begin{array}{l}10 \text { second hold } / 5 \\
\text { second rest }\end{array}$ \\
\hline $\begin{array}{l}\text { On all fours position + Hip, knee } \\
\text { extension + Plantar } \\
\text { flexion + Exercise band } \\
\text { (hip and + plantar flexion) }\end{array}$ & slow & $2 \times 10$ second & $4 \times 10$ second & $\begin{array}{l}10 \text { second hold } / 5 \\
\text { second rest }\end{array}$ \\
\hline
\end{tabular}

Table 2. Exercise protocol.

\begin{tabular}{|c|c|c|c|}
\hline \multicolumn{4}{|c|}{ Isotonic Exercises } \\
\hline & & \multicolumn{2}{|c|}{ Duration } \\
\hline & & Phase $1-4$ weeks & Phase 4 - 8 weeks \\
\hline Exercise & Tempo & Volume & Volume \\
\hline Wall squat $45^{\circ}$ & Slow & $2 \times 10$ rep & $4 \times 10$ rep \\
\hline Jumping from step in pairs & Slow & $2 \times 10$ rep & $4 \times 10$ rep \\
\hline $\begin{array}{l}\text { Hip and knee flexion/extension } \\
\text { using partner }\end{array}$ & Slow & $2 \times 10$ rep & $4 \times 10$ rep \\
\hline $\begin{array}{l}\text { Supine pushing the ball against the } \\
\text { wall, pushing the tiller }\end{array}$ & Slow & $2 \times 10$ rep & $4 \times 10$ rep \\
\hline Lunges $0^{\circ}-45^{\circ}$ flexion & Slow & $2 \times 10$ rep & $4 \times 10$ rep \\
\hline $\begin{array}{l}\text { On all fours position }+ \text { Hip, Knee } \\
\text { extension + Plantar flexion + Exercise } \\
\text { band (hip and knee 90-degree } \\
\text { extension + plantar flexion) }\end{array}$ & Slow & $2 \times 10$ rep & $4 \times 10$ rep \\
\hline
\end{tabular}

\subsection{Statistical Analyses}

In this study, first Smirnov-Kolmogorov was used to examine the normal distribution; then, parametric or non-parametric tests were used. Since data had a normal distribution, in order to compare the studied variables across the three experimental groups (two exercise-performing groups and a control group) from before to after the intervention, analysis of variance among participants was used for repeated measurements in a $2 \times 3$ plan ( 3 groups and 2 times). Bonferroni post hoc test was used to identify significant differences. The significance level was considered to be equal to 0.05 for all statistical tests. The SPSS statistical software (version 16) was also used for statistical calculations.

\section{Finding}

Descriptive mean and standard deviation obtained in this study included age, height, weight, and body mass index, which are shown in Table 3. The mean and standard deviation of Stork and YBT variables are shown in Table 4. The 
results of analysis of variance with repeated measures to compare the balance of the three groups are also reported in Table 5.

Table 3. Descriptive mean and standard deviation of age, height, weight, and BMI.

\begin{tabular}{cccccc}
\hline Variable & Isotonic & Isometric & Control & F & P \\
\hline Age (years) & $26.40 \pm 3.23$ & $27.70 \pm 2.40$ & $27.10 \pm 3.75$ & 0.41 & 0.66 \\
Mass (Kg) & $176.60 \pm 6.22$ & $178 \pm 6.73$ & $176.70 \pm 7.46$ & 0.13 & 0.87 \\
Height (cm) & $71.30 \pm 9.26$ & $73.40 \pm 8.40$ & $70.40 \pm 9.25$ & 0.29 & 0.74 \\
BMI & $22.71 \pm 1.54$ & $23.11 \pm 1.08$ & $22.47 \pm 1.34$ & 0.58 & 0.56
\end{tabular}

Table 4. Mean and standard deviation of stork and YBT.

\begin{tabular}{|c|c|c|c|c|c|}
\hline Variable & Direction & - & Isometric & Isotonic & Control \\
\hline & & Pre-test & $85.50 \pm 6.078$ & $85.3 \pm 6.074$ & $85.70 \pm 4.98$ \\
\hline & & Post-test & $90.30 \pm 7.43$ & $92.80 \pm 6.64$ & $85.20 \pm 4.58$ \\
\hline \multirow{4}{*}{ YBT } & & Pre-test & $87.10 \pm 7.14$ & $84.10 \pm 4.55$ & $84.60 \pm 4.50$ \\
\hline & & Post-test & $91.40 \pm 9.02$ & $90.90 \pm 4.09$ & $85.30 \pm 5.85$ \\
\hline & & Pre-test & $83.90 \pm 4.72$ & $87.90 \pm 6.60$ & $85.40 \pm 3.09$ \\
\hline & Posterolateral & Post-test & $88.70 \pm 5.37$ & $93.70 \pm 8.94$ & $87.20 \pm 1.93$ \\
\hline \multirow[t]{2}{*}{ Stork } & - & Pre-test & $11.87 \pm 2.93$ & $12.07 \pm 2.71$ & $11.76 \pm 2.44$ \\
\hline & & Post-test & $12.99 \pm 2.47$ & $13.21 \pm 2.46$ & $11.82 \pm 2.40$ \\
\hline
\end{tabular}

Table 5. Differences between isometric and isotonic exercises in terms of the two variables.

\begin{tabular}{|c|c|c|c|c|c|}
\hline Variable & Direction & Factor & $\mathrm{F}$ & $\mathrm{P}$ & Effect size \\
\hline & & Time & 78.03 & $0.001^{*}$ & 0.74 \\
\hline & Anterior & Group & 0.96 & 0.39 & 0.067 \\
\hline & & Time $^{\star}$ Group & 27.84 & $0.001^{*}$ & 0.67 \\
\hline & & Time & 40.71 & $0.001^{*}$ & 0.60 \\
\hline \multirow[t]{6}{*}{ YBT } & Posteromedial & Group & 1.53 & 0.27 & 0.091 \\
\hline & & Time $^{\star}$ Group & 8.24 & $0.002^{*}$ & 0.38 \\
\hline & & Time & 46.62 & $0.001^{*}$ & 0.63 \\
\hline & Posterolateral & Group & 2.35 & 0.11 & 0.14 \\
\hline & & Time $^{\star}$ Group & 3.94 & $0.031^{\star}$ & 0.22 \\
\hline & & Time & 44.20 & $0.001^{\star}$ & 0.62 \\
\hline \multirow[t]{2}{*}{ Stork } & - & Group & 0.30 & 0.74 & 0.022 \\
\hline & & Time $^{\star}$ Group & 9.33 & $0.001^{\star}$ & 0.41 \\
\hline
\end{tabular}

Bonferroni post hoc test results showed that after eight weeks of EMSII exercises of lower limbs, there was a significant increase in participants' static and 
dynamic balance. However, no significant difference was observed between isometric and isotonic exercises in terms of the two variables.

\section{Discussion}

It can be concluded from the results that the eight weeks of EMSII of lower extremity improve the static and dynamic balance of the participants in this study. This is in spite of the fact that no meaningful difference was observed suggesting any impact of these two types of exercises. It has been reported that improvement of physiological system and strengthening of weak muscles lead to an enhanced balance. Scientific evidence shows that the balance in individuals suffering from PFPS is inferior to that of healthy individuals [14] [18]. Since muscle weakness, lack of flexibility, and motor control problems are all responsible for poor balance, implementation of an appropriate exercise program is an effective strategy for improving balance [28]. Previous studies show that muscle weakness and disorder of the knee extensor mechanism are the cause of patella deviation from its original position, and consequently, pain in patients with PFPS [14] [29] [30]. Through changes in the balance reactions, individuals with PFPS choose positions without pain to keep their balance. This limits their balance performance [14] [31]. In addition, the visual awareness has an important role in maintaining postural stability. In general, it is reported that, by eliminating visual information, postural sway is increased by 50\% [32]. The study of Miser et al. showed that proprioception of the knee joint in patients with anterior knee pain syndrome was reduced compared to the control group [33]. In a study conducted by Ecopa et al. in 2005 on patients with the anterior cruciate ligament injury, it was observed that the level of postural sway in these individuals, when standing on the affected leg, increases with eyes closed. These researchers introduced the vision as an important factor in compensating for the anterior cruciate ligament (ACL) proprioceptive system dysfunction [34]. Therefore, according to the research conducted by Ecopa, Miser and Heath Kamp (2000), increased muscle strength can increase intra- and extra-muscular muscle coordination. In additionIn addition, muscle strength causes optimal activity and tension in agonist muscle, where it may be possible that the exercises presented in this study enhance static balance and proprioceptive sensory system [35].

Anoka believes that increase in muscle strength caused by appropriate exercises may result in changes in neural mechanisms, including increased output of supraspinal centers, changes in the neural mechanism that lead to reduced contraction activity in antagonist muscles, increased activity of agonist muscles, and effective inter-neuronal communication [36]. Therefore, it can be said that improved static balance is the result of muscle strengthening without any manipulation in the visual system. But as far as the static balance with closed eyes and eliminated "vision" are concerned, incoming messages from the eyes, as well as a proprioceptive sensory system in the legs, are affected. This is while EMSII of lower limbs could improve the static balance with closed eyes. 
One of the possible reasons for the significant increase in the balance of the participants in this study is the importance of the ankle strategy. In this strategy, muscles of the ankle, knee, and hip joint, as well as core muscles can reduce the arisen interactions, while keeping the center of gravity at the base of support and prevent instability, the interplay involving the ankle strategy can be effective in the creation of balance and its improvement [37]. However, fatigue and lack of sufficient muscle strength can also be considered important and influential in this strategy, and underscore the fact that isometric exercises-which were previously known as endurance training [38] - have the ability to increase muscle endurance. This characteristic can delay the muscle fatigue which is one of the main causes of imbalance [39].

The effect of single-leg squat exercise on dynamic balance cannot be ignored [40]. In the present study, this motion was implemented in the same way as the squat exercise, with this difference that relying on a wall squat by standing on both legs were allowed for additional support. It is likely that, by improving balance, these exercises can reduce the risk of falls is elite athletes, increase coordination in their functions, and generally lead to enhanced performance in executing their techniques. However, the improved balance can also be due to the positive effect of exercises on proprioceptive sensory receptors, and also the fact that the increased strength caused by the leg squat exercises has improved the dynamic balance. These results are in accordance with the results of the study conducted by Toole et al. (2000) on determining the effect of squats using weights on balance and mobility in standing position in patients with partial spinal cord injury. Tang et al. (2010) studied the effect of adding sitting and standing exercises to the movement therapy exercises and concluded that, the added exercises improved dynamic balance in patients with stroke, compared to the patients having only undergone movement therapy [41]. Since the movement therapy exercises used for the two groups are a sort of strength training intended to strengthen the muscles such as Iliopsoas muscle and the quadriceps, the movement therapy exercises were added to the experimental group and lead to improved balance in patients in this group.

In a study, Tok et al. (2009) examined the impact of isotonic exercises on the dynamic balance of people with arthritis. The exercises included electric stimulation combined with increased range of motion movements. The researchers observed that these exercises had a significant effect in improving balance [42].

\section{Study Limitation}

The majority of the factors affecting the variables were controlled in this research. However, several factors potentially affecting the variables could not be controlled by the researcher. These factors include: nutrition, motivation, genetic characteristics, amount and quality of sleep, lack of strict control over subjects' activities outside the research hours, small sample size, lack of access to Biodex, as well as lack of access to electromyography for evaluating muscle activity. 


\section{Conclusion}

At the end of this study, although participants did not fully recover from PFPS, they did show improved balance. Therefore, it seems that isometric and isotonic exercises had the desired effects in this regard. On the other hand, no difference was observed between the two types of exercises, which is probably due to the short period of practice or the limited variety of extensor muscle sling exercises of lower limbs. However, not a lot of studies have been conducted in this regard. This is perhaps one of the first studies that compare the impact of isometric and isotonic exercises on the static and dynamic balance among female basketball players suffering from patellofemoral pain syndrome.

\section{Acknowledgements}

The authors thank all participants and their kindly cooperation.

\section{References}

[1] Banan. Motarjem, Tina (1389) Compression of Hyper Mobility of Joints and Knee and Ankle Injuries in Female Athletes. Master's Thesis, University of Tehran, Tehran, 70-71. (Abstract in English)

[2] Lathinghouse, L.H. and Trimble, M.H. (2000) Effects of Isometric Quadriceps Activation on the Q-Angle in Women before and after Quadriceps Exercise. Journal of Orthopaedic \& Sports Physical Therapy, 30, 211-216. https://doi.org/10.2519/jospt.2000.30.4.211

[3] Letafatkar, A., Abdolvahabi, Z. and Rahmati, H. (2010) Reliability of Knee Functional Tests in Patients with Patellofemoral Pain Syndrome. Journal of Research in Rehabilitation Science, 5, 116-126. (Abstract in Persian).

[4] Witvrouw, E., Werner, S., Mikkelsen, C., Van Tiggelen, D., Berghe, L. V. and Cerulli, G. (2005) Clinical Classification of Patellofemoral Pain Syndrome: Guidelines for Non-Operative Treatment. Knee Surgery, Sports Traumatology, Arthroscopy, 13, 122-130. https://doi.org/10.1007/s00167-004-0577-6

[5] Douciette, S.A. and Goble, E.M. (1992) The Effect of Exercise on Patellar Tracking in Lateral Patellar Compression Syndrome. The American Journal of Sports Medicine, 20, 434-440. https://doi.org/10.1177/036354659202000412

[6] Duffey, M.J., Martin, D.F., Cannon, D.W., Craven, T. and Messier, S.P. (2000) Etiologic Factors Associated with Anterior Knee Pain in Distance Runners. Medicine and Science in Sports and Exercise, 32, 1825-1832. https://doi.org/10.1097/00005768-200011000-00003

[7] Eckhoff, D.G., Brown, A.W., Kilcoyne, R.F. and Stamm, E.R. (1997) Knee Version Associated with Anterior Knee Pain. Clinical Orthopaedics and Related Research, 339, 152-155. https://doi.org/10.1097/00003086-199706000-00020

[8] Eng, J.J. and Pierrynowski, M.R. (1993) Evaluation of Soft Foot Orthotics in the Treatment of Patellofemoral Pain Syndrome. Physical Therapy, 73, 62-68. https://doi.org/10.1093/ptj/73.2.62

[9] Avraham, F., Aviv, S., Ya'akobi, P., Faran, H., Fisher, Z., Goldman, Y. and Carmeli, E. (2007) The Efficacy of Treatment of Different Intervention Programs for Patellofemoral Pain Syndrome-A Single Blinded Randomized Clinical Trial. Pilot Study. The Scientific World Journal, 7, 1256-1262. https://doi.org/10.1100/tsw.2007.167 
[10] Bockrath, K., Wooden, C., Worrell, T., Ingersoll, C.D. and Farr, J. (1993) Effects of Patella Taping on Patella Position and Perceived Pain. Medicine \& Science in Sports \& Exercise, 25, 989-992. https://doi.org/10.1249/00005768-199309000-00003

[11] Hazneci, B., Yildiz, Y., Sekir, U., Aydin, T. and Kalyon, T.A. (2005) Efficacy of Isokinetic Exercise on Joint Position Sense and Muscle Strength in Patellofemoral Pain Syndrome. American Journal of Physical Medicine \& Rehabilitation, 84, 521-527. https://doi.org/10.1097/01.phm.0000167682.58210.a7

[12] Crossley, K., Bennell, K., Green, S., Cowan, S. and McConnell, J. (2002) Physical Therapy for Patellofemoral Pain a Randomized, Double-Blinded, Placebo-Controlled Trial. The American Journal of Sports Medicine, 30, 857-865. https://doi.org/10.1177/03635465020300061701

[13] Arndt, A., Westblad, P., Winson, I., Hashimoto, T. and Lundberg, A. (2004) Ankle and Subtalar Kinematics Measured with Intracortical Pins during the Stance Phase of Walking. Foot \& Ankle International, 25, 357-364. https://doi.org/10.1177/107110070402500514

[14] Kooroshfard, N., Alizadeh, M.H. and Kahrizi, S. (1388) Comparison of Dynamic Balance in Female Futsal Players with Patellofemoral Pain Syndrome and Healthy People. Journal of Sport Medicine, No. 2, 10-22.

[15] Frank, C. and Lardner, R. (2010) Assessment and Treatment of Muscle Imbalance: The Janda Approach. Human Kinetics, 60, 70.

[16] Ebrahimi, E., Salavati, M., Marufi, N. and Esmaili, V. (2007) The Effect of Balance Training on Equilibrium Tests and Dynamic Satability Index in Healthy Men by Biodex Stability System. Journal of Rehabilitation Medicine, 2, 19-25.

[17] Goto, S. (2009) The Effect of Ptellofemoral Pain Syndrome on the Hip and Knee Neuromuscular Control on Dynamic Postural Control Task. Doctoral Dissertation, University of Toledo.

[18] Aminaka, N. and Gribble, P.A. (2008) Patellar Taping, Patellofemoral Pain Syndrome, Lower Extremity Kinematics, and Dynamic Postural Control. Journal of Athletic Training, 43, 21-28. https://doi.org/10.4085/1062-6050-43.1.21

[19] Bennell, K., Wee, E., Crossley, K., Stillman, B. and Hodges, P. (2005) Effects of Experimentally-Induced Anterior Knee Pain on Knee Joint Position Sense in Healthy Individuals. Journal of Orthopaedic Research, 23, 46-53.

[20] Callaghan, M.J., Selfe, J., McHenry, A. and Oldham, J.A. (2008) Effects of Patellar Taping on Knee Joint Proprioception in Patients with Patellofemoral Pain Syndrome. Manual Therapy, 13, 192-199.

[21] Geiser, C., O'connor, K.M. and Earl, J.E. (2010) Effects of Isolated Hip Abductor Fatigue on Frontal Plane Knee Mechanics. Medicine \& Science in Sports \& Exercise, 42, 535-545.

[22] Citaker, S., Kaya, D. and Yukse, I. (2011) Static Balance in Patient with Patellofemoral Pain Syndrome. Sports Health, 3, 524-527.

[23] Román, P.Á.L., López, D.M., Fuentes, A.R. and Pinillos, F.G. (2017) Reference Values of Static Balance in Spanish Preschool Children. Perceptual and Motor Skills, 124, 740-753.

[24] Nia, M.H. and Zarei, A.M. (1392) Compression Static Balance in Active and Inactive Adult and Elderly Men. Salmand Journal, 15, 18.

[25] Plisky, P.J., Rauh, M.J., Kaminski, T.W. and Underwood, F.B. (2006) Star Excursion Balance Test as a Predictor of Lower Extremity Injury in High School Basketball Players. Journal of Orthopaedic \& Sports Physical Therapy, 36, 911-919. 
https://doi.org/10.2519/jospt.2006.2244

[26] Hrvvie, D., O’leavy, T. and Kuma, S. (2011) A Systematic Review of Randomize Controlled Trial on Exercise Parameters in Treatments of Patellofemoral Pain: What Works?

[27] Wisbey-Roth, T. (2012) Dynamic Slings and Optimal 3D Function. Olympic/Specialist Sports Physiotherapist (FACP), Masters of Sport Physiotherapy (AIS/UC) Active Rehabilitation Consultant, 5-8.

[28] Spiers, S. (2010) Comparison of the Effects of Aquatic and Land-Based Balance Training Programs on the Proprioception of College-Aged Recreational Athletes. Doctoral Dissertation.

[29] Binda, S.M., Culham, E.G. and Brouwer, B. (2003) Balance, Muscle Strength, and Fear of Falling in Older Adults. Experimental Aging Research, 29, 205-219. https://doi.org/10.1080/03610730303711

[30] Carter, N.D., Khan, K.M., Mallinson, A., Janssen, P.A., Heinonen, A., Petit, M.A. and McKay, H.A. (2002) Knee Extension Strength Is a Significant Determinant of Static and Dynamic Balance as well as Quality of Life in Older Community-Dwelling Women with Osteoporosis. Gerontology, 48, 360-368. https://doi.org/10.1159/000065504

[31] Stane, M.L. and Powers, M.E. (2005) The Effects of Plyometric Training on Selected Measures of Leg Strength and Power When Compared to Weight Training and Combination Weight and Plyometric Training. Journal of Athletic Training, 42, 186-192.

[32] Kawakita, T., Kuno, S., Miyake, Y. and Watanabe, S. (2000) Body Sway Induced by Depth Linear Vection in Reference to Central and Peripheral Visual Field. The Japanese Journal of Physiology, 50, 315-321. https://doi.org/10.2170/jjphysiol.50.315

[33] Messier, S.P., Glasser, J.L., Ettinger, W.H., Craven, T.E. and Miller, M.E. (2002) Declines in Strength and Balance in Older Adults with Chronic Knee Pain. Medicine \& Science in Sports \& Exercise, 34, S36. https://doi.org/10.1097/00005768-200205001-00206

[34] Okuda, K. (2005) Effect of Vision on Postural Sway in Anterior Cruciate Ligament Injured Knees.

[35] Heitkamp, H.C., Horstmann, T., Mayer, F., Weller, J. and Dickhuth, H.H. (2001) Gain in Strength and Muscular Balance after Balance Training. International Journal of Sports Medicine, 22, 285-290. https://doi.org/10.1055/s-2001-13819

[36] Enoka, R.M. (1997) Neural Strategies in the Control of Muscle Force. Muscle \& Nerve, 20, 66-69. https://doi.org/10.1002/(SICI)1097-4598(1997)5+<66::AID-MUS16>3.0.CO;2-X

[37] Jeon, S.N. and Choi, J.H. (2015) The Effects of Ankle Joint Strategy Exercises with and without Visual Feedback on the Dynamic Balance of Stroke Patients. Journal of Physical Therapy Science, 27, 2515-2518. https://doi.org/10.1589/jpts.27.2515

[38] Petrofsky, J.S., Burse, R.L. and Lind, A.R. (1975) Comparison of Physiological Responses of Women and Men to Isometric Exercise. Journal of Applied Physiology, 38, 863-868.

[39] Triscott, S., Gordon, J., Kuppuswamy, A., King, N., Davey, N. and Ellaway, P. (2008) Differential Effects of Endurance and Resistance Training on Central Fatigue. Journal of Sports Sciences, 26, 941-951. https://doi.org/10.1080/02640410701885439

[40] Toole, T., Hirsch, M.A., Forkink, A., Lehman, D.A. and Maitland, C.G. (2000) The 
Effects of a Balance and Strength Training Program on Equilibrium in Parkinsonism: A Preliminary Study. NeuroRehabilitation, 14, 165-174.

[41] Tung, F.L., Yang, Y.R., Lee, C.C. and Wang, R.Y. (2010) Balance Outcomes after Additional Sit-to-Stand Training in Subjects with Stroke: A Randomized Controlled Trial. Clinical Rehabilitation, 24, 533-542. https://doi.org/10.1177/0269215509360751

[42] Tok, F., Aydemir, K., Peker, F., Safaz, İ., Taşkaynatan, M.A. and Özgül, A. (2011) The Effects of Electrical Stimulation Combined with Continuous Passive Motion versus Isometric Exercise on Symptoms, Functional Capacity, Quality of Life and Balance in Knee Osteoarthritis: Randomized Clinical Trial. Rheumatology International, 31, 177-181. https://doi.org/10.1007/s00296-009-1263-2

Submit or recommend next manuscript to SCIRP and we will provide best service for you:

Accepting pre-submission inquiries through Email, Facebook, LinkedIn, Twitter, etc. A wide selection of journals (inclusive of 9 subjects, more than 200 journals) Providing 24-hour high-quality service User-friendly online submission system Fair and swift peer-review system Efficient typesetting and proofreading procedure Display of the result of downloads and visits, as well as the number of cited articles Maximum dissemination of your research work

Submit your manuscript at: http://papersubmission.scirp.org/ Or contact health@scirp.org 